Espaço Aberto | Open Space

\title{
Descolonizando a saúde planetária
}

\section{Decolonizing planetary health}

\author{
João Biehl' \\ https://orcid.org/0000-0003-4843-4804 \\ jbiehl@princeton.edu \\ I Princeton University - Princeton, NJ, Estados Unidos
}




\title{
Resumo
}

Intimamente conectada com decisões políticas e interesses de mercado, a pandemia de Covid-19 é uma calamidade crônica agudizada que assola o mundo inteiro, desestabilizando conhecimentos e práticas biomédicas hegemônicas e revelando a precariedade dos sistemas de saúde pública, assim como a impotência profunda das redes de seguridade social e a fragilidade dos laços de solidariedade que imaginávamos estáveis. $\mathrm{O}$ artigo reflete sobre os desafios impostos aos cientistas sociais e profissionais da saúde em contextos neoliberais e à beira da autocracia num momento em que seus métodos e conceitos-chave são chamados a dar conta de forma mais adequada aos complexos enredos territorializados pela emergência generalizada e a cultivar micro, meso e macromodos de resistência. Assim, instrumentos analíticos da antropologia médica e da saúde global crítica, como vulnerabilidade estrutural, determinantes políticos, racialização, farmaceuticalização e descolonização do saber, podem ser valiosos recursos para leitura do presente e de intervenção nele, mas são também desafiados pela dinâmica realidade que se desdobra. Contra o pano de fundo de uma crescente tensão entre a tecnocrática produção de microdispositivos humanitários e o desenvolvimento de uma ciência integrada de saúde planetária, o artigo ilumina a urgência da articulação de uma ética amazônica de cuidado.

Palavras-chave: saúde global crítica e antropologia médica; saúde planetária; descolonização; ética do cuidado.

\begin{abstract}
Intimately connected with political decisions and market concerns, the COVID-19 pandemic is an acute on chronic calamity that plagues the entire world, destabilizing hegemonic biomedical knowledges and practices and revealing the precariousness of public health systems as well as the deep impotence of social safety nets and the fragility of bonds of solidarity that we imagined existed. The article reflects on the challenges posed to social scientists and health professionals in neoliberal contexts and on the verge of autocracy when their methods and key concepts are being called to better account for the complex plots territorialized by the widespread emergency and to cultivate dynamic micro-meso-macro modes of resistance. Thus, analytical tools of medical anthropology and critical global health, such as structural vulnerability, political determinants, racialization, pharmaceuticalization and decolonization of knowledge, can be valuable resources for understanding the present and intervening in it, but are also challenged by the dynamically unfolding reality. Against the backdrop of growing tension between the technocratic production of humanitarian micro devices and the development of an integrated science of planetary health, the article illuminates the urgency of articulating an Amazonian ethics of care.
\end{abstract}

Keywords: critical global health and medical anthropology; planetary health; decolonization; ethics of care. 


\section{Mundos no limite}

Vivemos à beira do abismo. Esse presságio serviu de premissa a muitos trabalhos realizados em antropologia médica e estudos críticos de saúde global nas últimas duas décadas. A lógica se baseia nas evidências sobre a desigualdade, a violência e a precariedade que ameaçam vidas e sociedades, indicando que muitas delas estariam à beira do esgotamento. Tudo isso mediado pelo populismo extremo e pelos esforços estatais e empresariais para desmantelar agendas fragmentadas, embora significativas, de direitos socioeconômicos. Entrementes, etnografias atentas ao sensorial também lançaram luz sobre a plasticidade das pessoas e das comunidades, sobre seus desejos de mudança e rotas de fuga, enfatizando o poder de criação e agência da gente simples (Biehl, 2020; Biehl; Locke, 2017).

Os tempos do presságio ficaram para trás. Hoje, minha reflexão se faz em meio aos desdobramentos da tragédia. "Essa história acontece em estado de emergência e de calamidade pública", escreve Clarice Lispector (2017) no preâmbulo do seu último romance, $A$ hora da estrela, ecoando vivências que remetem às atuais. Ela segue: "Trata-se de um livro inacabado porque lhe falta a resposta. Resposta esta que espero que alguém no mundo me dê. Vós?"

A pandemia da Covid-19 pode ser pensada como um novo fato social total, como teorizou o antropólogo Marcel Mauss (1990, p. 80; Viveiros de Castro, 2020): fato esse que demanda respostas sem precedentes. Intimamente conectada a decisões políticas e interesses de mercado, a pandemia assola o mundo inteiro, desestabilizando conhecimentos e práticas biomédicas hegemônicas e revelando a precariedade dos sistemas de saúde pública, assim como a impotência profunda das redes de seguridade social e a fragilidade dos laços de solidariedade que imaginávamos estáveis. No meio de danos devastadores à saúde, à macro e microeconomia e ao próprio campo político, somos também confrontados com o impacto letal da devastação ambiental, com os limites dos nossos sistemas de prevenção e com as formas arraigadas de violência estrutural. Como temos visto, a Covid-19 continua a se espalhar e a matar de forma desigual em termos de idade, classe, raça, sexo e geografia (Biehl; Günay, 2020), especialmente em contextos de viés autoritário e de negação da ciência.

Saturados pela mídia social e mais divididos que nunca, a situação nos pede que depositemos o que resta de esperança nos fabricantes de vacinas, torcendo 
para que restaurem algum sentido de normalidade à vida social e política. Ao mesmo tempo tememos, é claro, que, diante da chegada das tecnologias de salvação, desigualdades se aprofundem e sistemas de controle se fortaleçam (Greene; Vargha, 2020).

Enquanto a vacinação demora, a questão racista chegou a ponto de ebulição, como vimos nos últimos meses aqui nos Estados Unidos, com a população negra e aliados enfrentando abertamente a supremacia branca e o racismo sistêmico que, durante séculos, limitaram e excluíram as oportunidades de vida dos não brancos, muitas vezes sob o pretexto da preservação da ordem política e do humanismo liberal e de sistemas falaciosos de responsabilização (Davis, 2003; Gilmore, 2007; Glaude, 2020; Ralph, 2020; Shange, 2019; Vitale, 2018).

No meio desse sentimento geral de vertigem e perante a promessa fugidia de reparação e abolição, perguntamo-nos de que formas a pandemia e os movimentos por justiça racial se conectam. Não que um necessariamente dependa do outro, mas imagino que esta crise global de saúde pública e as possibilidades de um mundo remodelado pós-Covid-19 inspirem vislumbres de esperança, de um outro horizonte ainda possível.

\section{Para além das intervenções tecnocráticas}

O que, então, o momento atual pede à antropologia, à medicina social e aos estudos críticos de saúde global? O que agora se exige da nossa escuta, da nossa produção de evidências e da nossa capacidade de resposta em termos de solidariedade concreta? Como atentar para vulnerabilidades, sofrimentos e experiências de iminência da morte, assim como a formas emergentes de política e de cuidado ainda que à distância? Nesse estado de emergência sem paralelo, pessoas em todo o mundo são impelidas a repensar as arquiteturas e pressupostos do capitalismo médico, do poder político e da vida social e econômica. Do mesmo modo, somos impelidos a repensar o nosso legado disciplinar, os nossos focos de investigação e o nosso papel público enquanto acadêmicos (Biehl, 2011; Biehl; Petryna, 2014).

A nossa tarefa torna-se ainda mais desafiadora diante das origens e legados coloniais das ciências sociais e da própria saúde global (Allen; Jobson, 2016; Asad, 1973; Biehl, 2016a; Kopenawa; Albert, 2013; Povinelli, 2002; Richardson, 2019; 
Said, 1989; Simpson, 2014; Smith, 2012; Trouillot, 2003). No seu melhor, tais campos prosperaram ao produzir conhecimento de forma relacional e situada e ao desestabilizar hierarquias de especialização; lograram ao utilizar uma análise historicamente atenta, abrindo também arquivos insurgentes; se destacaram ao se engajar em uma interlocução reflexiva com diversos profissionais que também procuraram desconcertar formas hegemônicas de pensamento sobre o humano, o ético e o político. Tais profissionais e interlocutores, assim como as nossas disciplinas, apontaram assim para a inter-relação do biológico com o social e com o meio ambiente, entendendo a saúde não como a faculdade de restauração da normalidade, mas como uma capacidade normativa de se adaptar em face da ameaça de morte - a saúde como um direito humano a ser exigido e concretizado (Adams, 2013; Biehl, 2007, 2013a; Bourgois; Schonberg, 2009; Briggs; Mantini-Briggs, 2016; Canguilhem, 1978; Das, 2015; Farmer, 2005; Farmer, et al., 2013; Fassin, 2007; Holmes, 2013; Petryna, 2013; Scheper-Hughes, 1991; Wendland, 2010; Whyte, 2015).

Contra o pano de fundo de uma pandemia em expansão e da luta intensificada contra o racismo sistêmico, o meu ensaio se engaja com a convicção de que a antropologia médica e a medicina preventiva são singularmente capazes de refletir sobre o momento que a saúde global vive. Isso é devido ao nosso compromisso etnográfico e ativista com o corpo plural e com distintas condições humanas, bem como à articulação de contribuições teóricas e práticas para a saúde pública e para a arte do cuidado. Engajados com mundos locais (Garcia, 2010; Han, 2012; Kleinman, 2006; Kleinman; Das; Lock, 1997) de maneira "historicamente profunda e geograficamente ampla" (Farmer, 2004, p. 309, tradução minha), realçamos a importância de examinar criticamente as formas de conhecimento que reproduzem e invisibilizam os mecanismos de exclusão, assim como os modos micro, meso e macro de resistência. Permanecemos atentos ao fato de que, como diria James Baldwin (1998, p. 723, tradução minha), "a história está literalmente presente em tudo o que fazemos"; mantemos, assim, os nossos modos de existência e interferência abertos à improvisação e à constante recalibração.

Nesse sentido, vale a pena considerar as críticas importantes que surgiram após a resposta tecnocrática "extremamente inadequada e tardia" (Farrar; Piot, 2014, p. 1545, tradução minha) da saúde global à epidemia de Ebola na África Ocidental de 2014 a 2016. Tal reação provocou disparidades grotescas 
em termos de riscos e consequências e, em retrospectiva, ignorou determinantes sociais e políticos que estavam no cerne da crise do Ebola (Biehl, 2016a; Richardson et al., 2017).

Uma primeira vertente crítica destacou a natureza neocolonial da saúde global, ou seja, a associação do global com o modus operandi euro-americano e os usos de emergências como oportunidades para todo o tipo de experimentações (Anderson, 2014; Cueto, 2007; Dahn; Mussa; Nutt, 2015). Uma segunda vertente explorou a forma como as respostas globais de saúde geralmente refletem e sustentam a ordem capitalista neoliberal, uma vez que as intervenções são canais de interesses geoeconômicos, cada vez mais sob a tutela de atores do setor privado (Adams, 2016; Birn, 2014; Birn; Dmitrienko, 2005; Keshavjee, 2014). Uma terceira vertente crítica adotou uma abordagem foucauldiana, centrando-se na emergência de novos regimes de biossecuritização que estão passando da prevenção à prontidão, criando assim novos modos de vigilância e encorajando respostas técnicas que pouco consideram as condições de vida locais (Lakoff, 2017; Lakoff; Collier, 2008; Lakoff; Collier; Kelty, 2015; Segata; Mastrangelo, 2020; Ventura, 2016). Um último aporte crítico viu a resposta global de saúde ao surto de Ebola como uma manifestação de humanitarismo transnacional, enfatizando a desigualdade e a violência que frequentemente acompanham a assistência (Fassin, 2011; Redfield, 2013; Ticktin; Feldman, 2010; Stevenson, 2014). O fato é que, no pós-epidemia, os limites da saúde global foram mais uma vez expostos na medida em que o financiamento e a atenção eram suspensos e realocados para outros fins - ainda que o sofrimento continuasse na forma de sequelas clínicas, perda de entes queridos, comunidades desfeitas, insegurança alimentar e estigma social (Cancedda et al., 2016; Frankfurter, 2019).

O que está em jogo, então, não é apenas a preparação tecnopolítica, mas também a preparação científico-social e o desafio de abraçar outra ética e mudanças "radicais de comportamento" na governança a nível global, como Richard Horton (2014, p. 2186, tradução minha), editor-chefe do The Lancet, então preconizou. Os estudos críticos da saúde global e os conceitos-chave da antropologia médica têm se revelado, nesse sentido, bastante pertinentes e relevantes em face das forças turbulentas que se desdobram à nossa volta, pressionando nossos métodos e análises a se aproximarem de forma mais adequada aos complexos enredos territorializados do nosso tempo (Biehl; Petryna, 2013; Biehl; Locke, 2017; Schuch; Víctora; Silva, 2018; Wilkinson; Kleinman, 2015). 


\section{Expondo vulnerabilidades estruturais e combatendo o racismo sistêmico}

Apesar de vivermos um momento por demais incerto, sabemos que a Covid-19 ilumina vulnerabilidades arraigadas em nossas sociedades (Diniz, 2016). Ela é um evento acute-on-chronic, ou crônico agudizado, como Paul Farmer (2012, p. 3) sintetizou ao se referir ao terremoto no Haiti em 2010 e à catástrofe humanitária que ocasionou, assim como ao surto de Ebola a que me referi. A vulnerabilidade estrutural é uma lente crítica que oferece explicação à propagação tão desigual de um vírus respiratório, que atinge mais gravemente grupos sub-representados, como que no encalço da estratificação social (Ayres; Paiva; França Jr., 2011; Farmer, 2006; Metzl; Hansen, 2014; Quesada; Hart; Bourgois, 2011).

Em sentido semelhante, também o conceito de racialização é fundamental para entendermos as desigualdades nas taxas de mortalidade pela Covid-19 e a sua codificação na literatura médica. Penso aqui em trabalhos como os de Carolyn Rouse (2009), Ruha Benjamin (2019) e Catherine Bliss (2012) nos Estados Unidos, mas também no que tem sido feito no Brasil. Há a produção crescente do grupo de trabalho em Racismo e Saúde da Abrasco, com nomes como os de Denize Ribeiro (2014) e Edna Maria de Araújo (Araújo et al., 2009, Araújo et al., 2015; Caldwell; Araújo, 2020; Hogan et al., 2018; Smolen et al., 2018), assim como os estudos ativistas de Jurema Werneck (2009, 2016; Werneck et al., 2013), Maria do Carmo Leal (2017) e Kia Lilly Caldwell (2004, 2017). O conceito de racialização e suas variantes ajudam a expor regimes de invisibilização e descaso. O combate à vulnerabilidade estrutural e à racialização requerem, como bem dizem Jonathan Metzl e Helena Hansen (2014), não uma competência cultural, mas uma competência estrutural por parte dos profissionais preocupados com uma saúde integral de fato.

Os paradigmas reinantes da farmaceuticalização e da privatização da saúde para os quais apontam meus trabalhos junto a pacientes psiquiátricos e pessoas vivendo com HIV/AIDS no Brasil (Biehl, 2007, 2008, 2013a, 2013b, 2016b, 2016c) revelam-se totalmente inadequados para lidar com as muitas facetas de uma resposta à pandemia (Griner, 2020). As inadequações se evidenciam na logística de testagem, no rastreio de contatos, na falta de envolvimento de comunidades, bem como na dificuldade de aumentar a capacidade hospitalar para cuidados intensivos em todas as regiões do país. Ainda assim, um 
complexo industrial pandêmico capitaliza o desastre (como diria a pensadora e ativista Naomi Klein [2008, 2020]) com promessas salvadoras tecnocráticas, baseadas em soluções mágicas de mercado.

Como nexo revelador de vulnerabilidades infraestruturais, a nova pandemia também colocou em nítido relevo a questão da casa como espaço físico, biossocial e afetivo relevante para a saúde pública, os itinerários terapêuticos e a economia (Biehl; Neiburg, 2021). Tornaram-se centrais, nesse contexto de pandemia, as preocupações antropológicas com a forma como os mundos da vida se fazem e refazem na relação com a casa e as configurações habitacionais, entendidas como entes relacionais (Carsten; Hugh-Jones, 1995; Cavalcanti, 2012; Marcelin, 1996; Neiburg, 2019). As casas, juntamente com a reproblematização de regimes de trabalho e cuidado e daquilo que é considerado essencial à vida, têm de certa forma determinado quem a pandemia fará viver ou deixará morrer.

\section{Contrapolíticas e artes do possível}

Junto ao escrutínio dos determinantes políticos da pandemia (Gore; Parker, 2019; Saúde..., 2020; Ventura, 2020) precisamos também atentar para resistências e formas de counter-politics, ou contrapolítica dentro do próprio Estado. É nesse contexto que quero mencionar algumas tendências que despontam em um estudo que ora conduzo sobre a judicialização da Covid-19, em colaboração com o epidemiologista Joseph Amon e o doutorando Lucas Prates (Biehl; Amon; Prates, 2021). Estamos usando vários bancos de dados, inclusive o boletim Direitos na Pandemia, organizado pela Deisy Ventura e colegas (Asano et al., 2020), além de entrevistas com diversos protagonistas.

A pandemia evidencia um fortalecimento do papel político do Poder Judiciário (Biehl, 2013a; Vianna et al., 1999). Tal fortalecimento já parecia estar em curso antes de 2020; agora, contudo, percebemos não só um acirramento da briga decisória entre o Judiciário e os outros poderes, mas também o Judiciário tomando as rédeas das grandes decisões políticas do país (Especialistas..., 2020; Freitas, 2020). Isso ficou claro tanto em âmbito federal (com os embates recentes entre o STF e o presidente Jair Bolsonaro) quanto nas bases de nossa democracia. Diante da dúvida sobre implementar ou não medidas de distanciamento social, por exemplo, uma solução comumente encontrada em estados e municípios 
foi a de terceirizar a decisão para a Justiça. Ouvimos de interlocutores que entre a opinião vinda "da [secretaria de] saúde" e a opinião vinda "do jurídico", prefeitos e governadores ficariam com o direito. Outro importante exemplo foram os vários casos em que juízes ao redor do país decidiram sobre a realocação de vagas de UTI - determinando, na prática, quem sobreviveria e quem morreria.

Também percebemos o Judiciário brasileiro como um campo de embate político ainda mais aberto e distinto do que antes. Distinto porque parece ter assumido um papel de crescente protagonismo num cenário em que a política tradicional se recusa ou não consegue dar respostas urgentes na área da saúde. Aberto porque se realçam a inconstância e a instabilidade decisórias nesse mesmo contexto, que exige uma dinamicidade para que a solução jurídica seja, ad hoc, a que melhor se adapte ao contexto político, econômico e de saúde pública. Há certamente divergências interpretativas importantes aqui: alguns autores, por exemplo, defendem que estamos vendo o Judiciário assumindo o seu papel de sempre, apenas aparando arestas democráticas (Fontainha; Castro, 2020). Estaria de fato o Judiciário se portando meramente como um contraponto político, ou estaria, na onda da pandemia, realizando uma contrapolítica desde um outro projeto de poder? E o que essa judicialização da política via pandemia diz sobre a saúde de nossa democracia e sobre os desafios de políticas públicas que se nos apresentam?

A pandemia também explicita os determinantes políticos do próprio Poder Judiciário, e vice-versa. Durante a crise da Covid-19, temos visto que o acesso à Justiça se encontra ainda mais restrito que o normal. Com muitas delegacias, defensorias públicas e outros órgãos de Justiça atendendo remotamente, pessoas sem acesso à internet e a equipamentos eletrônicos encontram mais uma grande barreira para registrar boletins de ocorrência, realizar consultas jurídicas, iniciar procedimentos judiciais, e assim por diante (Diniz; Medeiros, 2020; Soprana, 2020). Este parece ser mais um exemplo de que a casa e as configurações habitacionais são essenciais para a análise da pandemia e de suas consequências.

\section{"A pluralidade das formas de vida"}

Quero concluir com um breve comentário sobre dois modelos emergentes design thinking humanitário e saúde planetária (Biehl; Ong, 2019) - que estão impactando as formas como os atores da saúde global reinventam as suas 
agendas e intervenções. A saúde global hoje oscila entre soluções de escalas dramaticamente diferentes. Por um lado, há o foco no design de dispositivos de pequena escala destinados ao uso em situações de catástrofe ou extrema pobreza - como purificadores de água, kits de diagnóstico rápido, ou ainda monitores médicos portáteis (Brown; Wyatt, 2010; PATH, 2015; Redfield, 2016). Por outro, há o levantamento de novos recursos e aliados para enfrentar os riscos ambientais relacionados a mudanças geofísicas e ecológicas de larga escala (Brondizio et al., 2016; Horton et al., 2014; Rodin, 2015). É importante entender o que esses modelos produzem ao se assentarem em torno da Covid-19.

Enquanto a guinada para uma microssaúde global, como eu a chamo, pode atrair a atenção para ausências ou fraquezas do Estado, e refletir um desencanto com projetos de mudanças mais amplas, os cientistas sociais insistem - acho que com razão - que esses crescentes microdispositivos inovadores são, sob disfarce humanitário, o triunfo encoberto da lógica neoliberal e tecnocrática (Moran-Thomas, 2013; Schwittay; Braund, 2017). No caso do Brasil, podemos ver o "kit-Covid" propagandeado por Bolsonaro, incluindo a infame cloroquina (Collucci, 2020), como um exemplo de truque farmaceuticalizado, em vez de uma resposta nacional de saúde pública robusta e coordenada. É uma expressão clara da politização catastrófica das ações deste (des)governo. Enquanto o Brasil se torna um laboratório biopolítico para o desenvolvimento de vacinas contra a Covid-19, vemos a população brasileira adentrar uma ordem experimental (Petryna, 2009), literalmente vivendo na posição de cobaias (Andreoni; Londoño, 2020).

Um dos principais desafios para uma ciência mais integrada de saúde planetária, por sua vez, tem sido a ênfase em grandes escalas (Chakrabarty, 2012; Frumkin; Myers, 2017). Uma narrativa universalizante de risco ambiental desvia a atenção do que conhecemos sobre os perigos ambientais reais, muitas vezes desigualmente distribuídos e afetando desproporcionalmente comunidades socioeconomicamente vulneráveis (Brulle; Pellow, 2006; Nading, 2017; Singer; Hasemann; Raynor, 2016). A narrativa de grande escala pode também ofuscar as economias políticas particulares que destroem a natureza e perturbam a vida animal - fenômenos que estão no cerne das pandemias recentes, todas oriundas da transmissão animal-humana, a exemplo do HIV, do Ebola, da Zika e, agora, da Covid-19. Num evento recente do Brazil LAB (Covid-19 and Amazonia's future, 2020), o virologista Pedro Vasconcelos falou que mais de 200 arbovírus 
já foram identificados por cientistas na Amazônia, e que o feroz desmatamento atualmente em curso pode causar novos cruzamentos virais e potenciais surtos de novas doenças. Ou seja: árvores em pé previnem pandemias (A Terra..., 2020).

É, pois, imperativo aprender com a sabedoria cultural dos guardiões de longa data da floresta, à medida que articulamos esforços para salvaguardar a Amazônia, para o Brasil e para o planeta. E, no entanto, o coronavírus se espalha entre os povos indígenas em ritmo alarmante, como discute de forma comovente a antropóloga Aparecida Vilaça (2020) em seu livro Morte na floresta. Dados do Instituto Socioambiental (ISA) mostram que no final de agosto de 2020 já havia 27 mil casos confirmados entre 155 povos no Brasil, somando cerca de 700 mortes (Covid-19 e os povos indígenas, 2020). Mais vulneráveis à pandemia, povos indígenas têm dificuldade de acesso aos serviços de saúde, seja pela distância geográfica ou pela indisponibilidade ou insuficiência de equipes de cuidado - sem mencionar que, junto com o vírus, chegam também os invasores de terra e os garimpeiros.

"Temos que abandonar o antropocentrismo," diz o líder indígena, ambientalista e escritor Ailton Krenak (2020), "há muita vida além da gente, não fazemos falta na biodiversidade". Num ensaio recente, Krenak (2020) enfatiza que:

O vírus não mata pássaros, ursos, nenhum outro ser, apenas humanos. Quem está em pânico são os povos humanos e seu mundo artificial, seu modo de funcionamento que entrou em crise... Esse pacote chamado de humanidade vai sendo descolado de maneira absoluta desse organismo que é a Terra, vivendo numa abstração civilizatória que suprime a diversidade, nega a pluralidade das formas de vida, de existência e de hábitos.

\section{Criar outras histórias}

E assim voltamos ao presente ameaçado e à ética do cuidado que os tempos nos exigem. Há muito por fazer. Qual é, então, o nosso papel, como estudiosos, professores, escritores, ativistas e cuidadores ao interferir nesses mundos no limite - e, ao neles interferir, vermos nossos próprios conceitos e compromissos transformados? Como pode a saúde crítica global permanecer conectada a processos sociais em andamento? 
Em outras palavras, e baseando-me no conceito de "horizoning" - horizontar ou prospectar horizontes, da antropóloga Adriana Petryna (2015, 2017): como podemos esboçar, ainda com equipamentos inadequados, novos caminhos e projeções de chegada à medida que tentamos remodelar a saúde pública e os campos médico, científico e político? Pergunto não só na esteira da Covid-19, mas também no que se refere ao reconhecimento das múltiplas precariedades, descasos e mesmo possibilidades de extinção que caracterizam e materializam nossos tempos.

Horizontar pode nos ajudar a imaginar como desenvolver capacidades humanas e institucionais que transcendam a repetição da história oficial e que ajudem a defender, num espírito de abertura política radical, o que o saudoso economista e humanista Albert Hirschman (1971, p. 37, tradução minha) chamava de "o direito inalienável de cada pessoa e nação a um futuro não projetado".

Não escutemos os tiranos ou os pregadores do apocalipse, mas aqueles e aquelas que, como Krenak, tentam compartilhar a mensagem de um outro mundo possível: "Temos de parar de vender o amanhã", diz o líder indígena. “Tomara que não voltemos à normalidade, pois, se voltarmos, é porque não valeu nada a morte de milhares de pessoas no mundo inteiro" (Krenak, 2020).

É hora de escutar, contar e criar outras histórias. ${ }^{1}$

1 Agradeço a Lucas Prates e a Arbel Griner por seu engajamento crítico com este ensaio e por sua perspicaz e generosa ajuda editorial. Agradeço também a José Ricardo Ayres, Deisy Ventura, Richard Parker, Joseph J. Amon, Miquéias Mugge e Adriana Petryna por estimulantes discussões. 


\section{Referências}

ADAMS, V. Markets of sorrow, labors of faith: New Orleans in the wake of Katrina. Durham: Duke University Press, 2013.

ADAMS, V. Metrics: what counts in global health. Durham: Duke University Press, 2016.

ALLEN, J. S.; JOBSON, R. C. The decolonizing generation: (race and) theory in anthropology since the eighties. Current Anthropology, Chicago, v. 57, n. 2, p. 129-148, 2016.

ANDERSON, W. Making global health history: the postcolonial worldliness of biomedicine. Social History of Medicine, Oxford, v. 27, n. 2, p. 372-384, 2014.

ANDREONI, M.; LONDOÑO, E. Coronavirus crisis has made Brazil an ideal vaccine laboratory. The New York Times, New York, 15 Aug. 2020. Disponível em: https://www. nytimes.com/2020/08/15/world/americas/brazil-coronavirus-vaccine.html. Acesso em: 10 set. 2020 .

ARAÚJO, E. M. de et al. A utilização da variável raça/cor em Saúde Pública: possibilidades e limites. Interface (Botucatu), Botucatu, v. 13, n. 31, p. 383-394, dez. 2009.

ARAÚJO, E. M. de. et al. Apresentação dossiê 'Saúde da População Negra'. Revista $A B P N$, [s. l.], v. 7, p. 12-15, 2015.

ASAD, T. Anthropology and the colonial encounter. Berkshire: Ithaca Press, 1973.

ASANO, C. L. et al. (ed.). Direitos na pandemia: monitoramento e análise das normas jurídicas de resposta à Covid-19 no Brasil. São Paulo: Conectas Direitos Humanos: Centro de Pesquisas e Estudos sobre Direito Sanitário, n. 1, 8 jul. 2020. Disponível em: https://www.conectas.org/publicacoes/download/boletim-direitos-na-pandemia-nol. Acesso em: 10 set. 2020.

AYRES, J. R. C. M.; PAIVA, V.; FRANÇA JR., I. From natural history of disease to vulnerability: changing concepts and practices in contemporary public health. In: PARKER, R.; SOMMER, M. (ed.). Routledge handbook in global public health. Abingdon: Taylor and Francis, 2011. p. 98-107.

BALDWIN, J. The white man's guilt. In: BALDWIN, J. Baldwin: collected essays. New York: Library of America, 1998. p. 722-727.

BENJAMIN, R. Race after technology: abolitionist tools for the new Jim Code. Cambridge: Polity, 2019.

BIEHL, J. Will to live: AIDS therapies and the politics of survival. Princeton: Princeton University Press, 2007. 
BIEHL, J. Antropologia do devir: psicofármacos - abandono social - desejo. Revista de Antropologia, São Paulo, v. 51, n. 2, p. 413-449, 2008.

BIEHL, J. Antropologia no campo da saúde global. Horizontes Antropológicos, Porto Alegre, ano 17, n. 35, p. 257-296, jan./jun. 2011.

BIEHL, J. Vita: life in a zone of social abandonment (updated with a new afterword and photo essay). Berkeley: University of California Press, 2013a.

BIEHL, J. The judicialization of biopolitics. American Ethnologist, Arlington, v. 40, n. 3, p. 419-436, 2013b.

BIEHL, J. Theorizing global health. MAT: Medicine Anthropology Theory, Amsterdam, v. 3, n. 2, p. 127-142, 2016a.

BIEHL, J. Patient-citizen-consumers: the judicialization of health and the metamorphosis of biopolitics. Lua Nova, São Paulo, n. 98, p. 77-105, 2016b.

BIEHL, J. The Postneoliberal fabulation of power: on statecraft, precarious infrastructures, and public mobilization in Brazil. American Ethnologist, Arlington, v. 43, n. 3, p. 437-450, 2016c.

BIEHL, J. Do incerto ao inacabado: uma aproximação com a criação etnográfica. Mana, Rio de Janeiro, v. 26, n. 3, e263206, 2020.

BIEHL, J; AMON, J.; PRATES, A. Supreme Court v. necropolitics: the chaotic judicialization of COVID-19 in Brazil. Health and Human Rights Journal, Cambridge, v. 23, n. 2, 2021. No prelo.

BIEHL, J; GÜNAY, O. How to teach anthropology in a pandemic?. Somatosphere, [s. l.], 25 May 2020. Disponível em: http://somatosphere.net/2020/how-to-teach-anthropology-in-a-pandemic.html/. Acesso em: 10 set. 2020.

BIEHL, J; LOCKE, P. Ethnographic sensorium. In: BIEHL, J.; LOCKE, P. (ed.). Unfinished: the anthropology of becoming. Durham: Duke University Press, 2017. p. 1-38.

BIEHL, J.; NEIBURG, F. Oikography: ethnographies of house-ing in critical times. Cultural Anthropology, Arlington, v. 36, n. 4, 2021. No prelo.

BIEHL, J; ONG, Y. From global health to planetary and micro global health: theorising global health's present remodeling and scaling. In: PARKER, R. G.; GARCIA, J. (ed.). Handbook on the politics of global public health. New York: Routledge, 2019. p. 63-78. 
BIEHL, J; PETRYNA, A. Critical global health. In: BIEHL, J.; PETRYNA, A. (ed.). When people come first: critical studies in global health. Princeton: Princeton University Press, 2013. p. 1-22.

BIEHL, J; PETRYNA, A. Peopling global health. Saúde e Sociedade, São Paulo, v. 23, n. 2, p. 376-389, 2014.

BIRN, A.-E. Philanthrocapitalism, past and present: the Rockefeller Foundation, the Gates Foundation, and the setting(s) of the international/global health agenda. Hypothesis, [s. l.], v. 12, n. 1, e8, 2014.

BIRN, A.-E.; DMITRIENKO, K. The World Bank: global health or global harm?. American Journal of Public Health, Washington, DC, v. 95, n. 7, p. 1091-1092, 2005.

BLISS, C. Race decoded: the genomic fight for social justice. Stanford: Stanford University Press, 2012.

BOURGOIS, P.; SCHONBERG, J. Righteous dopefiend. Berkeley: University of California Press, 2009.

BRIGGS, C. L.; MANTINI-BRIGGS, C. Tell me why my children died: rabies, indigenous knowledge, and communicative justice. Durham: Duke University Press, 2016.

BRONDIZIO, E. S. et al. Re-conceptualizing the anthropocene: a call for collaboration. Global Environmental Change, [s. l.], v. 39, p. 318-327, 2016.

BROWN, T.; WYATT, J. Design thinking for social innovation. Development Outreach, Washington, DC, v. 12, n. 1, p. 29-43, 2010.

BRULLE, R. J.; PELLOW, D. N. Environmental justice: human health and environmental inequalities. Annual Review of Public Health, Palo Alto, v. 27, p. 103-124, 2006.

CALDWELL, K. L. Negras in Brazil: re-envisioning black women, citizenship, and the politics of identity. New Brunswick: Rutgers University Press, 2004.

CALDWELL, K. L. Health equity in Brazil: intersections of gender, race, and policy. Champaign: University of Illinois Press, 2017.

CALDWELL, K. L.; ARAÚJO, E. M. de. COVID-19 is deadlier for black Brazilians, a legacy of structural racism that dates back to slavery. The Conversation, [s. l.], 10 June 2020. Disponível em: https://heconversation.com/covid-19-is-deadlier-for-black-brazilians-a-legacy-of-structural-racism-that-dates-back-to-slavery-139430. Acesso em: 12 set. 2020 . 
CANCEDDA, C. et al. Strengthening health systems while responding to a health crisis: lessons learned by a nongovernmental organization during the Ebola virus disease epidemic in Sierra Leone. The Journal of Infectious Diseases, Chicago, v. 214, supl. 3, p. S153-S163, 2016.

CANGUILHEM, G. On the normal and the pathological. Traduzido por C. Fawcett. Editado por R. S. Cohen. Boston: D. Reidel, 1978.

CARSTEN, J.; HUGH-JONES, S. About the house: Lévi-Strauss and beyond. Cambridge: Cambridge University Press, 1995.

CAVALCANTI, M. From shack to house to fortress: memory, space and favela consolidation in contemporary Rio de Janeiro. Oxford: Wiley-Blackwell, 2012.

CHAKRABARTY, D. Postcolonial studies and the challenge of climate change. New Literary History, Baltimore, v. 43, n. 1, p. 1-18, 2012.

COLLUCCI, C. Uso de 'kit Covid', sem eficácia comprovada, gera confronto entre médicos. Folha de S. Paulo, São Paulo, 10 jul. 2020. Disponível em: https://www1. folha.uol.com.br/equilibrioesaude/2020/07/uso-de-kit-covid-sem-eficacia-comprovada-gera-confronto-entre-medicos.shtml. Acesso em: 10 set. 2020.

COVID-19 AND AMAZONIA'S FUTURE. In: BRAZIL LAB. Princeton: Princeton University, 22 Apr. 2020. Disponível em: https://brazillab.princeton.edu/news/covid-19-and-amazonias-future. Acesso em: 10 set. 2020.

COVID-19 E OS POVOS INDÍGENAS. [S. l.]: Instituto Socioambiental, 2020. Disponível em: https://covid19.socioambiental.org/. Acesso em: 10 set. 2020.

CUETO, M. Cold war, deadly fevers: malaria eradication in Mexico 1955-1975. Baltimore: Johns Hopkins University Press, 2007.

DAHN, B.; MUSSA, V.; NUTT, C. Yes, we were warned about Ebola. The New York Times, New York, 8 Apr. 2015. Disponível em: https://www.nytimes.com/2015/04/08/opinion/yes-we-were-warned-about-ebola.html. Acesso em: 6 mar. 2018.

DAS, V. Affliction: health, disease, poverty. New York: Fordham University Press, 2015.

DAVIS, A. Are prisons obsolete?. New York: Penguin Random House, 2003.

DINIZ, D. Zika: do sertão nordestino à ameaça global. Rio de Janeiro: Civilização Brasileira, 2016.

DINIZ, D.; MEDEIROS, M. Acesso à tecnologia e garantia de direitos sociais. [S. l.]: Instituto Eduardo Correia, 23 jul. 2020. 1 vídeo (71min). Webinar promovido pelo Instituto Eduardo Correia (IEDC). Disponível em: https://www.youtube.com/ watch?v=q9QGVRfOEt0. Acesso em: 10 set. 2020. 
ESPECIALISTAS veem justiça entre excessos e necessidade de atuação em pandemia. CNN Brasil, São Paulo, 8 maio 2020. Disponível em: https://www.cnnbrasil. com.br/politica/2020/05/08/especialistas-veem-justica-entre-excessos-e-necessidade-de-atuacao-em-pandemia. Acesso em: 10 set. 2020.

FARMER, P. An anthropology of structural violence. Current Anthropology, Chicago, V. 45 , n. 3, p. 305-325, 2004.

FARMER, P. Pathologies of power: health, human rights, and the new war on the poor. Berkeley: University of California Press, 2005.

FARMER, P. Never again?: reflections on human values and human rights. Salt Lake City: University of Utah Press, 2006. (The Tanner Lectures on Human Values, v. 26).

FARMER, P. Haiti after the earthquake. New York: Public Affairs, 2012.

FARMER, P. et al. Introduction: a biosocial approach. In: FARMER, P. et al. (ed.). Reimagining global health: an introduction. Berkeley: University of California Press, 2013. p. 1-14.

FARRAR, J.; PIOT, P. The Ebola emergency: immediate action, ongoing strategy. The New England Journal of Medicine, Boston, v. 371, p. 1545-1546, 2014.

FASSIN, D. When bodies remember: experiences and politics of AIDS in South Africa. Berkeley: University of California Press, 2007.

FASSIN, D. Humanitarian reason: a moral history of the present. Berkeley: University of California Press, 2011.

FONTAINHA, F.; CASTRO, A. C. Justiça da pandemia: de volta ao 'passivismo' judicial?. Jota, Brasília, 4 set. 2020. Disponível em: https://www.jota.info/opiniao-e-analise/colunas/judiciario-e-sociedade/justica-pandemia-passivismo-judicial-04092020. Acesso em: 10 set. 2020.

FRANKFURTER, R. Conjuring biosecurity in the post Ebola Kissi Triangle: the magic of paperwork in a frontier clinic. Medical Anthropology Quarterly, v. 33, p. 517-538, 2019.

FREITAS, H. Toffoli: STF atuou como elemento estabilizador da ordem política, econômica e social. Jota, Brasília, 1 jul. 2020. Disponível em: https://www.jota.info/stf/ do-supremo/toffoli-stf-atuou-como-elemento-estabilizador-da-ordem-politica-economica-e-social-01072020. Acesso em: 10 set. 2020.

FRUMKIN, H.; MYERS, S. Health at a planetary scale: why we should think bigger about public health - way bigger. Politico, [s. l.], 13 Sept. 2017. Disponível em: https:// www.politico.com/agenda/story/2017/09/13/planetary-health-challenges-000514. Acesso em: 10 set. 2020. 
GARCIA, A. The pastoral clinic: addiction and Dispossession along the Rio Grande. Berkeley: University of California Press, 2010.

GILMORE, R. W. Golden gulag: prisons, surplus, crisis, and opposition in globalizing California. Berkeley: University of California Press, 2007.

GLAUDE, E. Begin again: James Baldwin's America and its urgent lessons for our own. New York: Penguin Random House, 2020.

GORE, R.; PARKER, R. Analysing power and politics in health policies and systems. Global Public Health, Abingdon, v. 14, n. 4, p. 481-488, 2019.

GREENE, J. A.; VARGHA, D. How epidemics end. In: BOSTON REVIEW. [S. l.: s. n.], 30 June 2020. Disponível em: http://bostonreview.net/science-nature/jeremy-greene-dora-vargha-how-epidemics-end. Acesso em: 10 set. 2020.

GRINER, A. Pandemia, farmacologia e biopoder - ou sobre meu encontro com o SARS-CoV2 e Rosa B. In: BVPS: Blog da Biblioteca Virtual do Pensamento Social. [S. l.]: BVPS, 22 maio 2020. Disponível em https://blogbvps.wordpress.com/2020/05/22/ pandemia-farmacologia-e-biopoder-ou-sobre-meu-encontro-com-o-sars-cov2-e-rosa-b-por-arbel-griner/. Acesso em: 10 set. 2020.

HAN, C. Life in debt: times of care and violence in neoliberal Chile. Berkeley: University of California Press, 2012.

HIRSCHMAN, A. O. A bias for hope: essays on development and Latin America. New Haven: Yale University Press, 1971.

HOGAN, V. K. et al. "We black women have to kill a lion everyday?": an intersectional analysis of racism and social determinants of health in Brazil. Social Science \& Medicine, Oxford, v. 199, p. 96-105, 2018.

HOLMES, S. Fresh fruit, broken bodies. Berkeley: University of California Press, 2013.

HORTON, R. Offline: can Ebola be a route to nation-building?. The Lancet, [s. l.], v. 384, n. 9961, p. 2186, 2014.

HORTON, R. et al. From public to planetary health: a manifesto. The Lancet, [s. l.], v. 383, n. 9920, p. 847, 2014.

KESHAVJEE, S. Blind spot: how neoliberalism infiltrated global health. Berkeley: University of California Press, 2014.

KLEIN, N. The shock doctrine: the rise of disaster capitalism. New York: Picador, 2008. 
KLEIN, N. Coronavirus capitalism - and how to beat it. The Intercept, [s. l.], 16 Mar. 2020. Disponível em: https://theintercept.com/2020/03/16/coronavirus-capitalism/. Acesso em: 10 set. 2020.

KLEINMAN, A. What really matters: living a moral life amidst uncertainty and danger. Oxford: Oxford University Press, 2006.

KLEINMAN, A.; DAS, V.; LOCK, M. (ed.). Social suffering. Berkeley: University of California Press, 1997.

KOPENAWA, D.; ALBERT, B. The falling sky: words of a Yanomami shaman. Cambridge: Harvard University Press, 2013.

KRENAK, A. O amanhã não está à venda. São Paulo: Companhia das Letras, 2020. Ebook.

LAKOFF, A. Unprepared: global health in a time of emergency. Oakland: University of California Press, 2017.

LAKOFF, A.; COLLIER, S. Biosecurity interventions: global health and security in question. New York: Columbia University Press, 2008.

LAKOFF, A. COLLIER, S.; KELTY, C. (ed.). Limn, [s. l.], n. 5, Ebola's ecologies, Jan. 2015. Disponível em: https://limn.it/issues/ebolas-ecologies/. Acesso em: 10 set. 2020.

LEAL, M. do C. A cor da dor: iniquidades raciais na atenção pré-natal e ao parto no Brasil. Cadernos de Saúde Pública, Rio de Janeiro, v. 33, supl. 1, p. 1-17, 2017.

LISPECTOR, C. A hora da estrela: edição com manuscritos e ensaios inéditos. Rio de Janeiro: Rocco Digital, 2017. Ebook.

MARCELIN, L. H. A invenção da família afro-americana. 1996. Tese (Doutorado em Antropologia) - Museu Nacional, Universidade Federal do Rio de Janeiro, Rio de Janeiro, 1996.

MAUSS, M. The gift: the form and reason for exchange in archaic societies. London: Routledge, 1990.

METZL, J. M.; HANSEN, H. Structural competency: theorizing a new medical engagement with stigma and inequality. Social Science \& Medicine, Oxford, v. 103, p. 126-133, 2014.

MORAN-THOMAS, A. A salvage ethnography of the Guinea worm: witchcraft, oracles and magic in a disease eradication program. In: BIEHL, J.; PETRYNA, A. (ed.). When people come first: critical studies in global health, Princeton: Princeton University Press, 2013. p. 207-242. 
NADING, A. Local biologies, leaky things, and the chemical infrastructure of global health. Medical Anthropology, Abingdon, v. 36, n. 2, p. 141-156, 2017.

NEIBURG, F. Conversas etnográficas haitianas. Rio de Janeiro: Papéis Selvagens, 2019. PATH. The IC2030 report: reimagining global health. [S. l.]: PATH, 2015. Disponível em: https://path.azureedge.net/media/documents/APP_ic2030_fs.pdf. Acesso em: 10 set. 2020.

PETRYNA, A. When experiments travel: clinical trials and the global search for human subjects. Princeton: Princeton University Press, 2009.

PETRYNA, A. Life exposed: biological citizens after Chernobyl. Princeton: Princeton University Press, 2013.

PETRYNA, A. What is a horizon? Navigating thresholds in climate change uncertainty. In: RABINOW, P.; SAMIMIAN-DARASH, L. (ed.). Modes of uncertainty: anthropological cases. Chicago: The University of Chicago Press, 2015. p. 147-164.

PETRYNA, A. Horizoning. In: BIEHL, J.; LOCKE, P. (ed.). Unfinished: the anthropology of becoming. Durham: Duke University Press, 2017. p. 243-269.

POVINELLI, E. The cunning of recognition: indigenous alterities and the making of Australian multiculturalism. Durham: Duke University Press, 2002.

QUESADA, J.; HART, L.; BOURGOIS, P. Structural vulnerability and health: Latino migrant laborers in the United States. Medical Anthropology, Abingdon, v. 30, n. 4, p. 339-362, 2011.

RALPH, L. The torture letters: reckoning with police violence. Chicago: The University of Chicago Press, 2020.

REDFIELD, P. Life in crisis: the ethical journey of Doctors Without Borders. Berkeley: University of California Press, 2013.

REDFIELD, P. Fluid technologies: the bush pump, the LifeStraw ${ }^{\circledR}$ and microworlds of humanitarian design. Social Studies of Science, London, v. 46, n. 2, p. 159-183, 2016.

RIBEIRO, D. A. Saúde coletiva e as demandas da população negra: desafios e perspectivas. In: SOUZA, M. K. B. de; TAVARES, J. S. (org.). Saúde coletiva e as demandas da população negra: desafios e perspectivas. Cruz das Almas: EDUFRB, 2014. v. 1, p. 105-115.

RICHARDSON, E. T. On the coloniality of global public health. Medicine Anthropology Theory, Amsterdam, v. 6, n. 4, p. 101-118, 2019. 
RICHARDSON, E. T. et al. The Ebola suspect's dilemma. The Lancet Global Health, [s. l.], v. 5, n. 3, e254-256, 2017.

RODIN, J. Planetary health: a new discipline in global health. In: THE ROCKEFELLER FOUNDATION. [S. l.]: The Rockefeller Foundation, 16 July 2015. Disponível em: https://www.rockefellerfoundation.org/blog/planetary-health-a-new-discipline-in-global-health/. Acesso em: 10 set. 2020.

ROUSE, C. Uncertain suffering: racial health care disparities and sickle cell disease. Berkeley: University of California Press, 2009.

SAID, E. Representing the colonized: anthropology's interlocutors. Critical Inquiry, Chicago, v. 15, n. 2, p. 205-225, 1989.

SAÚDE Global e COVID-19=Global Health and COVID-19. Princeton: Brazil LAB, 21 Aug. 2020. 1 vídeo (140min). Publicado no canal Brazil LAB at Princeton University. Disponível em: https://www.youtube.com/watch?v=R5LDAQOaNAw\&feature=emb_ logo\&ab_channel=BrazilLABatPrincetonUniversity. Acesso em: 10 set. 2020.

SCHEPER-HUGHES, N. Death without weeping: the violence of everyday life in Brazil. Berkeley: University of California Press, 1991.

SCHUCH, P.; VÍCTORA, C. G.; SILVA, S. B. da. As políticas de inclusão como problemática de engajamento antropológico. Horizontes Antropológicos, Porto Alegre, ano 24, n. 50, p. 7-24, jan./abr. 2018.

SCHWITTAY, A.; BRAUND, P. Iterate, experiment, prototype. Limn, [s. l.], n. 9, Nov. 2017. Disponível em: https://limn.it/articles/iterate-experiment-prototype/. Acesso em: 10 set. 2020 .

SEGATA, J.; MASTRANGELO, A. As biosseguranças e suas antropologias. Horizontes Antropológicos, Porto Alegre, ano 26, n. 57, p. 7-25, maio/ago. 2020.

SHANGE, S. Progressive dystopia: abolition, antiblackness, and schooling in San Francisco. Durham: Duke University Press, 2019.

SIMPSON, A. Mohawk interruptus: political life across the borders of settler states. Durham: Duke University Press, 2014.

SINGER, M.; HASEMANN, J.; RAYNOR, A. 'I feel suffocated': understandings of climate change in an inner-city heat island. Medical Anthropology, Abingdon, v. 35, n. 6, p. 453-463, 2016.

SMITH, L. T. Decolonizing methodologies: research and indigenous peoples. London: Zed Books, 2012. 
SMOLEN, J. R. et al. Intersectionality of race, gender, and common mental disorders in Northeastern Brazil. Ethnicity and Disease, [s. l.], v. 28, n. 3, p. 207-214, 2018.

SOPRANA, P. 70 milhões de brasileiros têm acesso precário à internet na pandemia do coronavírus. Folha de S. Paulo, São Paulo, 16 maio 2020. Disponível em: https:// www1.folha.uol.com.br/mercado/2020/05/cerca-de-70-milhoes-no-brasil-tem-acesso-precario-a-internet-na-pandemia.shtml. Acesso em: 10 set. 2020.

STEVENSON, L. Life beside itself: imagining care in the Canadian Arctic. Berkeley: University of California Press, 2014.

A TERRA é redonda: desnorteados. Piauí, São Paulo, 2 jun. 2020. Disponível em: https://piaui.folha.uol.com.br/terra-e-redonda-desnorteados/. Acesso em: 10 set. 2020.

TICKTIN, M.; FELDMAN, I. (ed.). In the name of humanity: the government of threat and care. Durham: Duke University Press, 2010.

TROUILLOT, M.-R. Anthropology and the savage slot: the poetics and politics of otherness. In: TROUILLOT, M.-R. Global transformations: anthropology and the modern world. New York: Palgrave Macmillan, 2003. p. 7-28.

VENTURA, D. From Ebola to Zika: international emergencies and the securitization of global health. Cadernos de Saúde Pública, Rio de Janeiro, v. 32, n. 4, e00033316, 2016.

VENTURA, D. Between science and populism: Brazil and COVID-19. In: AMERICAS' GLOBAL ROLE. London: Chatham House, 2 Apr. 2020. Disponível em: https://americas.chathamhouse.org/article/between-science-and-populism-brazil-and-covid-19/. Acesso em: 10 set. 2020.

VIANNA, L. W. et al. A judicialização da política e das relações sociais no Brasil. Rio de Janeiro: Editora Revan, 1999.

VILAÇA, A. Morte na floresta. São Paulo: Todavia, 2020.

VITALE, A. The end of policing. New York: Verso, 2018.

VIVEIROS DE CASTRO, E. Ce qui se passe au Brésil relève d'un génocide. Philosophie Magazine, Paris, 19 mai 2020. Disponível em: https://www.philomag.com/lactu/ temoignages/eduardo-viveiros-de-castro-ce-qui-se-passe-au-bresil-releve-dun-genocide-43226. Acesso em: 10 set. 2020.

WENDLAND, C. A heart for the work: journeys through an African medical school. Chicago: The University of Chicago Press, 2010. 
WERNECK, J. Nossos passos vêm de longe! Movimentos de mulheres negras e estratégias políticas contra o sexismo e o racismo. In: VERSCHUUR, C. (dir.). Vents d'Est, vents d'Ouest: mouvements de femmes et féminismes anticoloniaux. Genève: Graduate Institute Publications, 2009. p. 151-163. Disponível em: http://books.openedition.org/iheid/6316. Acesso em: 10 set. 2020.

WERNECK, J. Racismo institucional e saúde da população negra. Saúde e Sociedade, São Paulo, v. 25, n. 3, p. 535-548, 2016. Disponível em: https://doi.org/10.1590/S0104129020162610. Acesso em: 10 set. 2020.

WERNECK, J. et al. Racismo institucional: uma abordagem conceitual. [S. l.]: Geledés, 2013. Texto produzido para o Projeto Mais Direitos e Mais Poder para as Mulheres Brasileiras. Disponível em: https://www.geledes.org.br/wp-content/uploads/2013/05/FINAL-WEB-Racismo-Institucional-uma-abordagem-conceitual.pdf. Acesso em: 10 set. 2020.

WHYTE, S. R. (ed.). Second chances: surviving AIDS in Uganda. Durham: Duke University Press, 2015.

WILKINSON, I.; KLEINMAN, A. A passion for society: how we think about human suffering. Berkeley: University of California Press, 2015.

Recebido: 14/09/2020 Aceito: 20/01/2021 | Received: 9/14/2020 Accepted: 1/20/2021 